\title{
Gamma Knife Surgery for AVM
}

\author{
Lutfi Hendriansyah
}

\subsection{Symptoms and Signs}

Arteriovenous malformations (AVM) are an abnormal connection between arteries and veins lacking intervening capillary network characterized by a complex, tangled web of abnormal vessels and believed to be congenital although not hereditary. This abnormal tangled web of vessels is called nidus (Latin word for nest), which has abnormally high blood flow resulting from the absence of dampening effect of capillary and has high tendency to rupture resulting in stroke. Lack of capillary network also induces enlargement of especially venous side with subsequent "growth" of AVM resulting in mass effect to surrounding brain parenchyma. AVM also disrupts normal metabolism of surrounding brain tissue by depriving them of adequate circulation (steal phenomena) causing neurological symptoms depending on its location whether superficial in cortex or deep within thalamus, basal ganglia, corpus callosum, insula/sylvian fissure, brainstem, or cerebellum. These mechanisms stated above underlie pathogenesis of symptoms and signs including hemorrhage as the most common presenting symptom in $50-52 \%$ of cases, followed by seizure in $27-30 \%$, headache in $5-14 \%$, and progressive neurological deficit in less than
$5 \%[1,2]$. Hemorrhage is associated with high morbidity and mortality as much as $35 \%$ and $29 \%$, respectively, that it becomes a primary concern in AVM management. Overall annual risk of hemorrhage on untreated AVM ranges between 2 and $4 \%$, and although multiple studies implicated factors such as nidus size, complexity of AVM morphology, deep brain location or draining vein, associated aneurysm, and younger age to be related to hemorrhagic events, multivariate analysis indicated that only hemorrhage in initial presentation, deep AVM location or deep draining vein, and large nidus size is to be consistently predictive of higher risk of subsequent rupture up to $15 \%$ of annual rates $[1,2]$. Figure 32.1 shows presentation of AVM hemorrhage on brain computed tomography (CT) scan and angiogram.

AVM management includes treatment such as microsurgical resection, Gamma Knife surgery, and embolization either individually or in combination, or just simple clinical observation. The option between doing treatment and observation or among any treatment modality has to be carefully weighed according to risk-benefit ratio and must be balanced against the risk from natural course of the disease. This chapter focuses on Gamma Knife surgery as treatment modality for AVM, which has to be considered as treatment for selected patients.
L. Hendriansyah
Gamma Knife Center Indonesia, Siloam Hospitals
Lippo Village, Tangerang, Indonesia

(C) The Author(s) 2019

J. July, E. J. Wahjoepramono (eds.), Neurovascular Surgery, https://doi.org/10.1007/978-981-10-8950-3_32 


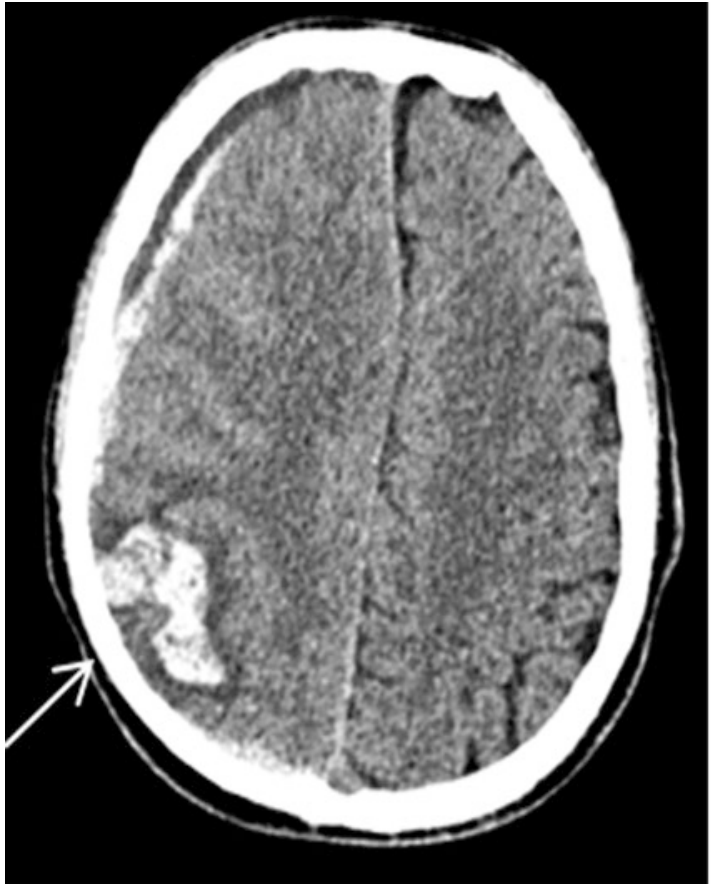

Fig. 32.1 Hemorrhage as AVM initial presentation. Spontaneous intracerebral hemorrhage in children or young adolescent, without history of hypertension and in uncommon location for hypertensive hemorrhage, should raise suspicion of AVM as the primary cause. CT

\subsection{Gamma Knife Surgery}

Gamma Knife surgery is commonly used to treat AVM that carry high risk of morbidity for microsurgical resection such as Spetzler-Martin grade III or above because it has the advantage of accurately treating small and deep-seated lesions with minimal risk of injury to surrounding structures. It delivers multiple fine beams of gamma rays radiation in such a highly focused manner that it can induce changes in AVM structure over time resulting in cessation of blood flow through the abnormal vessels leading to occlusion. The pathological changes following Gamma Knife surgery for AVM includes endothelial cell damage, proliferation of smooth muscle cells, and elaboration of extracellular collagen that subsequently lead to luminal stenosis and obliteration of nidus. Once complete obliteration of nidus happens, there will be no more blood flow through abnormal vessels. Overall total oblitera-

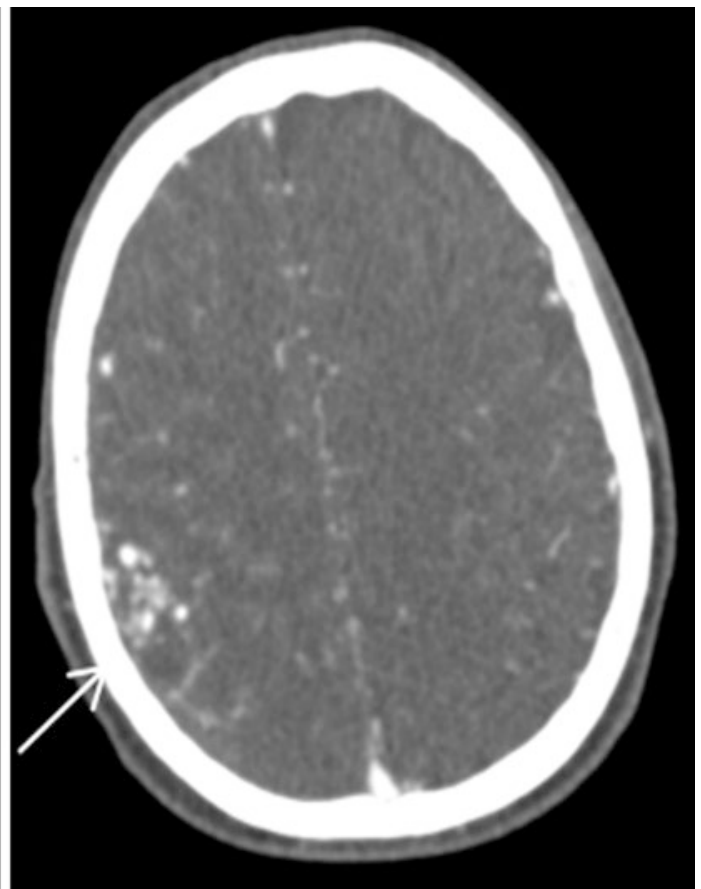

scan (left, white arrow) showing hemorrhage and CT angiogram (right, white arrow) demonstrating nidus anterior to bleeding site are two investigations that reveal hemorrhage as the most common initial presentation of AVM

tion rates of AVM following Gamma Knife surgery ranges between 72 and $85 \%$ in a typical single-session treatment, and this process usually takes 2-3 years to yield in result. High chance of cure together with low risk of morbidity makes this treatment modality an interesting option for AVM.

The aim of treatment is to accomplish total obliteration of AVM in which situation the risk of hemorrhage is alleviated and the patient is cured. In other situation where partial or subtotal obliteration occur, the risk of hemorrhage still persists and hence subsequent Gamma Knife treatment has to be considered. In predicting outcome after Gamma Knife surgery for AVM, several grading scales were developed such as Virginia Radiosurgery AVM Scale (VRAS) and radiosurgery-based grading AVM Score (RBAS), which includes into consideration history of hemorrhage and patient age, respectively, beside nidus volume and location. These grading scales 
are considered better in predicting favorable outcome after Gamma Knife surgery, compared to previously and more commonly used SpetzlerMartin Scale [3].

\subsection{Investigation}

If brain AVM is suspected, magnetic resonance imaging (MRI) can much better visualize AVM structure than CT scan. High-resolution MRI not only demonstrates AVM structures mainly nidus and draining veins, but it also shows the AVM location and its relation to surrounding cortical structures that are highly essential in setting up treatment planning. Digital subtraction angiography (DSA) is still the gold standard in visualizing AVM because this imaging can best demonstrate flow through every part of AVM in addition to structure. Combination of both imaging is important in defining the lesion for treatment and subsequently influencing optimal result in Gamma Knife surgery for AVM (Fig. 32.2).

\subsection{Preoperative Preparation}

In our institution, we use Leksell Gamma Knife ${ }^{\circledR}$ Perfexion $^{\mathrm{TM}}$ machine (Fig. 32.3, left) that is consisted of two main units; one is in radiation side and the other is in patient side. The radiation unit contains cobalt ${ }^{60}$ source that constantly emits gamma rays which is delivered to lesions during treatment through channel opening of 4,8 , or $16 \mathrm{~mm}$ collimators. Gamma rays are focused and accurately beamed to lesions through 192 collimator channels in which the opening and closing are controlled automatically according to treatment plan (Fig. 32.3, right). The patient side contains automated patient positioning system (PPS) that consists of a treatment couch where the patient lies down and of which movement is controlled according to treatment plan in such a way that lesion to be treated is positioned at the center of gamma rays cross-firing.

Leksell ${ }^{\circledR}$ Coordinate System utilizing titanium Leksell ${ }^{\circledR}$ G Frame as stereotactic frame is used to accurately localize lesion for treatment planning and to fixate the head as well as lesion in desired

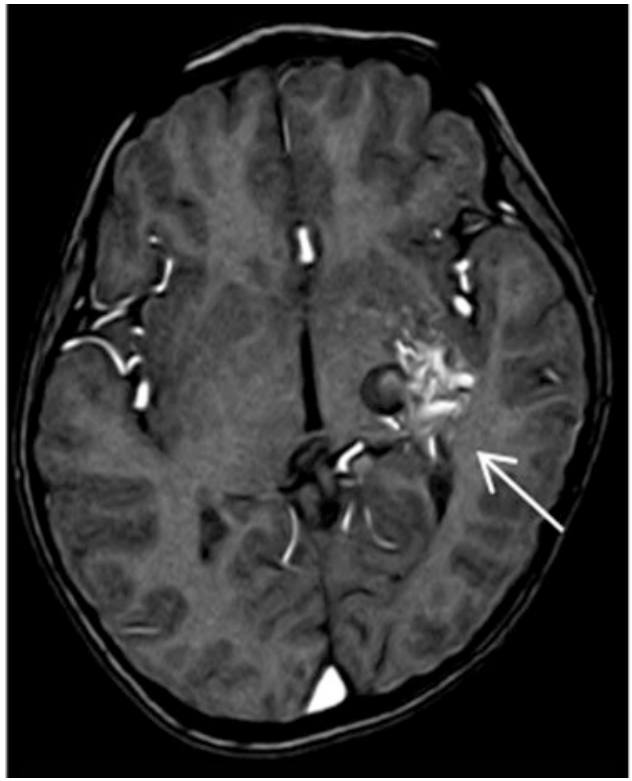

Fig. 32.2 MRI and DSA of AVM. T1 Fast Field Echo (FFE) MR images (left, white arrow) and DSA (right, black arrow) visualizing AVM nidus on posterior limb of

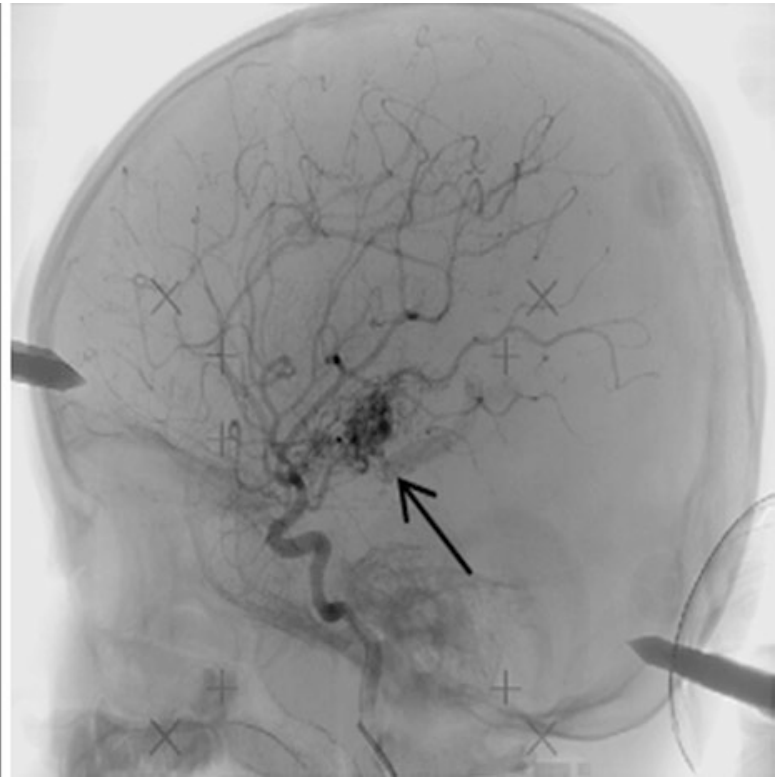

left internal capsule. Arterial feeder comes from medial branch of lenticulostriate artery and drains into Sylvian veins 

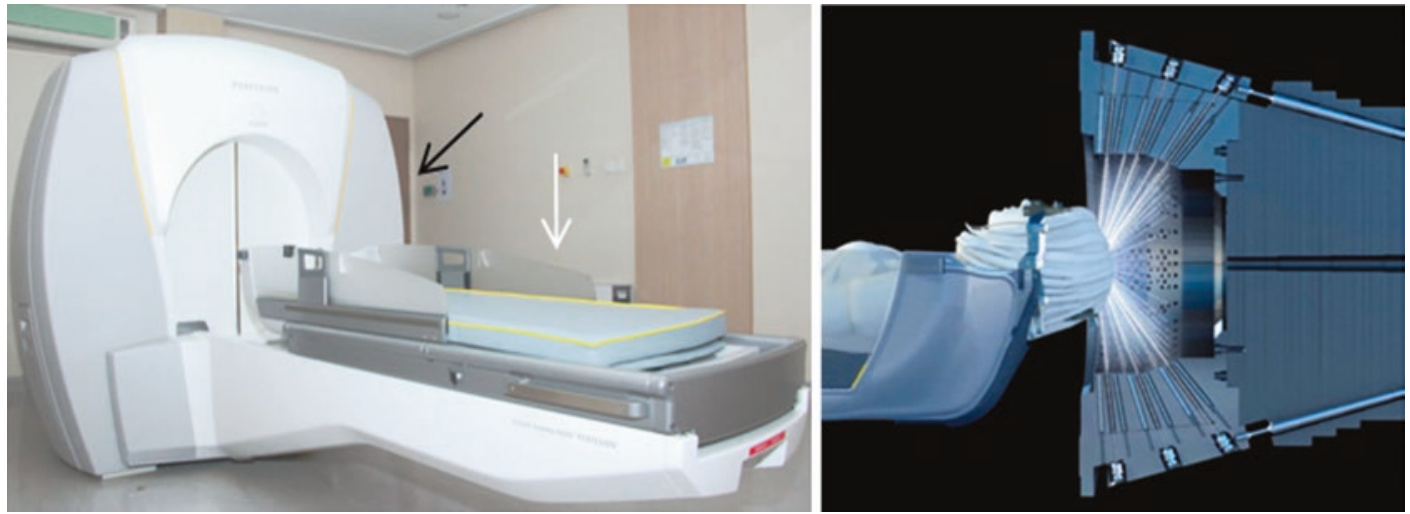

Fig. 32.3 Gamma Knife unit. Leksell Gamma Knife ${ }^{\circledR}$ Perfexion $^{\mathrm{TM}}$ unit. Left: radiation unit on the left half part of the machine (black arrow) and treatment couch on the

position during treatment delivery. It mainly consists of rectangular frame base, front piece, and a set of fixation posts and screws. Straight or curved fixation posts and screws measuring 20-110 $\mathrm{mm}$ are selected accordingly to fit different patient cranial shapes and sizes and hence to optimize operative field (Fig. 32.4). Frame is assembled during treatment preparation just before fixation to patient.

\subsection{Approach}

Gamma knife treatment plan uses several terminologies that every neurosurgeon needs to be familiar with in order to comprehend the treatment process. They are summarized in Table 32.1.

\subsection{Steps of the Surgery}

Treatment process begins with stereotactic frame fixation under local anesthesia (Fig. 32.5, left). General anesthesia is rarely used and only needed for children or those who are really uncooperative during treatment procedure. Leksell ${ }^{\circledR}$ G Frame is assembled and adjusted according to patients' head size and lesion location; two assistants help in positioning the frame, while the surgeon infiltrates local anesthesia into four right half part (white arrow). Right: Gamma ray delivery during treatment (courtesy of Elekta Co)

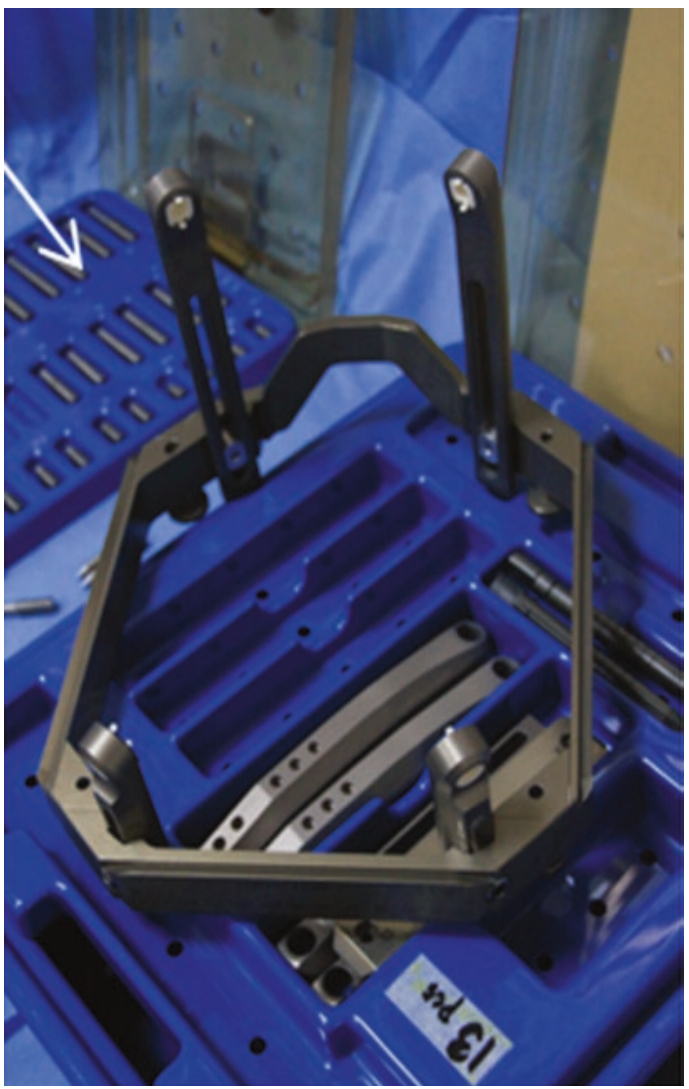

Fig. 32.4 Leksell ${ }^{\circledR} \mathrm{G}$ stereotactic frame. Frame consisting of rectangular frame base, curved front piece, and four fixation posts has been assembled and ready for placement. A set of fixation screws of different lengths is selected from tray (upper left, white arrow) 
Table 32.1 Terminology in Gamma Knife surgery

\begin{tabular}{|c|c|}
\hline Terminology & Description \\
\hline Target volume & Volume of lesion that is defined as target. In AVM it represents nidus volume \\
\hline Isocenter & $\begin{array}{l}\text { Point where radiation beams are converged by using combination of different collimators. } \\
\text { Isocenters are placed inside target volume }\end{array}$ \\
\hline Isodose line & $\begin{array}{l}\text { A line indicating distribution volume of same radiation dose. It is three-dimensional and is } \\
\text { composed by combination of isocenters. For example, } 12 \text { gray (Gy) isodose line demonstrates } \\
\text { nidus volume receiving } 12 \text { Gy }\end{array}$ \\
\hline Prescription isodose & $\begin{array}{l}\text { Isodose line of prescribed effective radiation dose that covers target volume. It is stated in } \\
\text { margin or peripheral dose. For example, margin dose of } 12 \mathrm{~Gy} \text { in } 50 \% \text { isodose means that } \\
\text { nidus margin is covered by } 12 \mathrm{~Gy} \text {, which is } 50 \% \text { of maximum dose }\end{array}$ \\
\hline Conformity index & $\begin{array}{l}\text { An index that compare isodose volume (prescription) inside target with total target volume. It } \\
\text { demonstrates degree to which target is covered by prescription dose and greater index reflects } \\
\text { more prescription dose inside the nidus }\end{array}$ \\
\hline Selectivity index & $\begin{array}{l}\text { An index that compare isodose volume (prescription) inside target with total isodose volume. } \\
\text { It demonstrates degree to which prescription dose is specific to target and do not spill to the } \\
\text { adjacent neurovascular structure. Greater index reflects less prescription dose outside the nidus }\end{array}$ \\
\hline Gradient index & $\begin{array}{l}\text { An index comparing prescription isodose with half value of this isodose. It reflects the } \\
\text { sharpness of radiation dose falloff around target margin, and smaller index demonstrates } \\
\text { steeper dose falloff outside the nidus }\end{array}$ \\
\hline
\end{tabular}
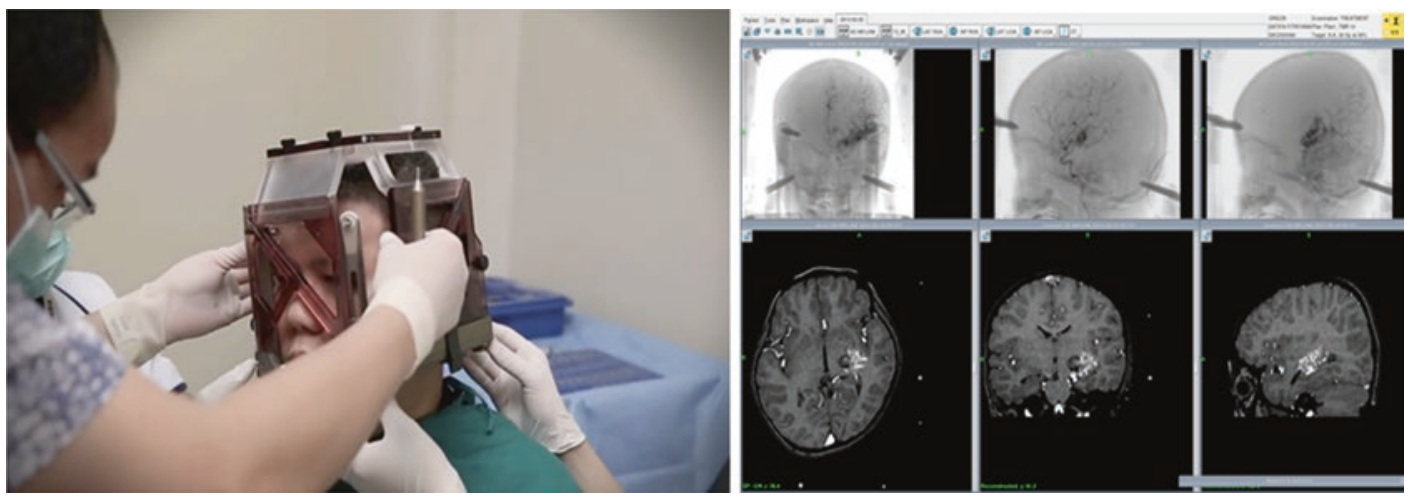

Fig. 32.5 Frame fixation and image acquisition. Sometimes MR indicator box is used during frame fixation to ensure adequate frame placement that covers opti-

points of pin insertion. A combination of lidocaine and adrenaline diluted with sterile water for injection is used for local anesthesia. As soon as anesthesia works, pins are tightened against the cranium, and frame position is checked to see whether it has fit the cranium properly or whether there is distortion caused by unequal pin pressure or excessive bending of titanium posts. Frame needs to be properly fit to cranium in order for treatment to reach lesions optimally, especially those with extremely eccentric location, and to avoid collision to collimator channel wall during treatment delivery. mal operative field (left). A combination of images from biplanar DSA and three-dimensional MR images is used for optimal nidus visualization (right)

When the frame is fixed, patients are ready for image acquisition (Fig. 32.5, right). Stereotactic DSA and MRI images are used for target localization. DSA is performed under local anesthesia; anteroposterior (AP) and lateral projections of feeding arteries, nidus, and draining veins are always sought for through both carotid and vertebrobasilar system bilaterally.

This way, every part of nidus can be visualized optimally and unlikely to be missed. It is essential to have optimal nidus visualization for maximum nidus coverage in treatment planning. The best images are then picked out for nidus delinea- 


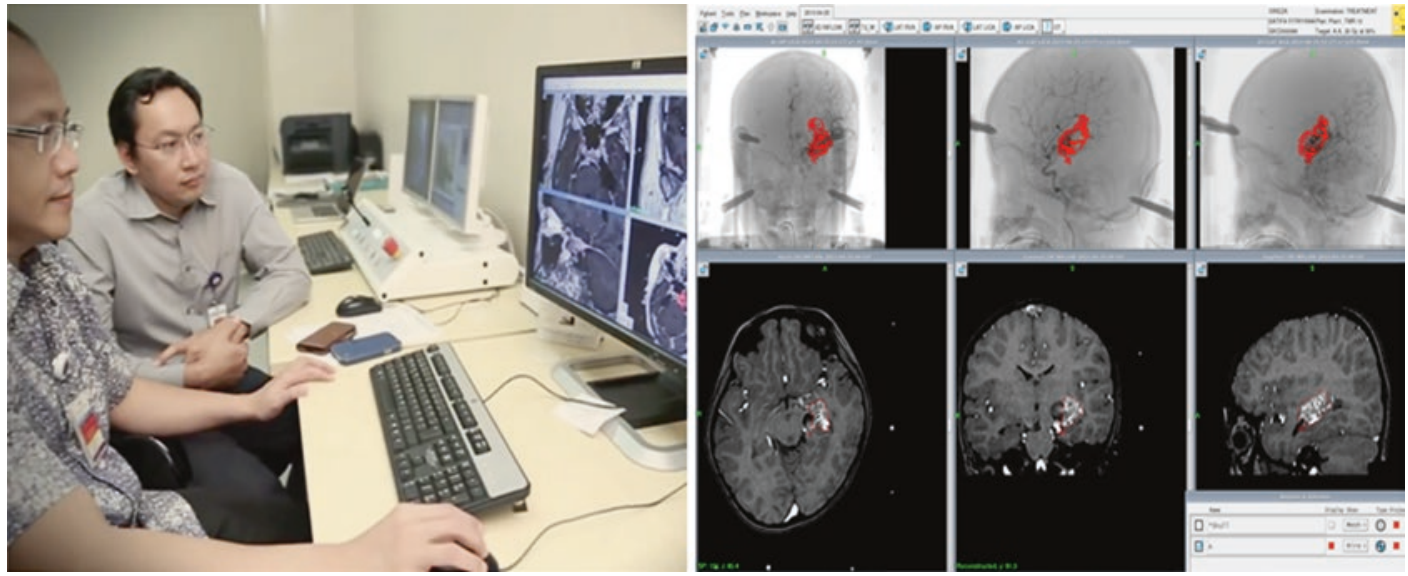

Fig. 32.6 Treatment planning and target delineation. Treatment is meticulously programmed on treatment planning system (TPS) using Gamma Plan ${ }^{\circledR}$ software. The whole nidus is defined as target volume and delineated (red line)

tion in order to define target volume, usually at the moment when the contrast agent starts leaving the nidus and filling up draining veins. In order not to miss this particular moment, DSA is set at minimum six frames per second (fps). Too late in taking images may result in overestimation of nidus size while too early may result otherwise. Since DSA images are made in two dimensions, they alone are not adequate to define stereotactic coordinates for accurate target localization in treatment planning that threedimensional MRI images are needed. We use 1.5 Tesla MRI with thin slice of $1 \mathrm{~mm}$-thickness without gap or overlap and usually without contrast injection. T2-weighted images together with T1 Fast Field Echo (FFE) technique are used to visualize the malformed vessels. Some patients cannot undergo MRI for many clinical reasons including severe claustrophobia and implantation of cardiac pacemakers. In these situations, threedimensional CT angiogram is used in defining stereotactic coordinates for lesion.

As soon as images are ready, they are then exported to the Gamma Knife treatment planning system (TPS). Gamma Plan ${ }^{\circledR}$ software in TPS is used to incorporate stereotactic DSA and MRI or CT images for target volume definition and delineation (Fig. 32.6).

After nidus is defined and well delineated, isocenter placement of prescribed treatment dose is initialized using a combination of 4,8 , or $16 \mathrm{~mm}$ collimators, to form prescription isodose line that best conform target lesion (Fig. 32.7). Margin dose ranging $18-25$ Gy in $40-50 \%$ isodose is commonly used for AVM treatment. There are several indices used to indicate how optimal treatment plan is. Conformity index, which indicates treatment coverage, is kept above 0.95 (95\% coverage), while selectivity index, which reflects how selective treatment is to target and thus avoid injuring surrounding healthy tissue, is kept above 0.92 (92\% selective to target), and gradient index, which reflects radiation spill outside target, is kept minimum below 3 .

As soon as treatment plan is finalized, the protocol is approved and exported from TPS into control system, which will control the whole operation of Gamma Knife unit during treatment delivery. Patient is then prepared for treatment.

Patient is positioned as comfortable as possible in treatment couch; frame is fixated to its dockings, and vital sign monitors are attached; treatment plan is rechecked and confirmed during time-out period before procedure (Fig. 32.8, left). Treatment plan has been approved in control system and ready for implementation (Fig. 32.8, right). Treatment then can be initialized by pressing start button in control system. We use closed-circuit TV (CCTV) camera with speakers and microphones inside treatment 


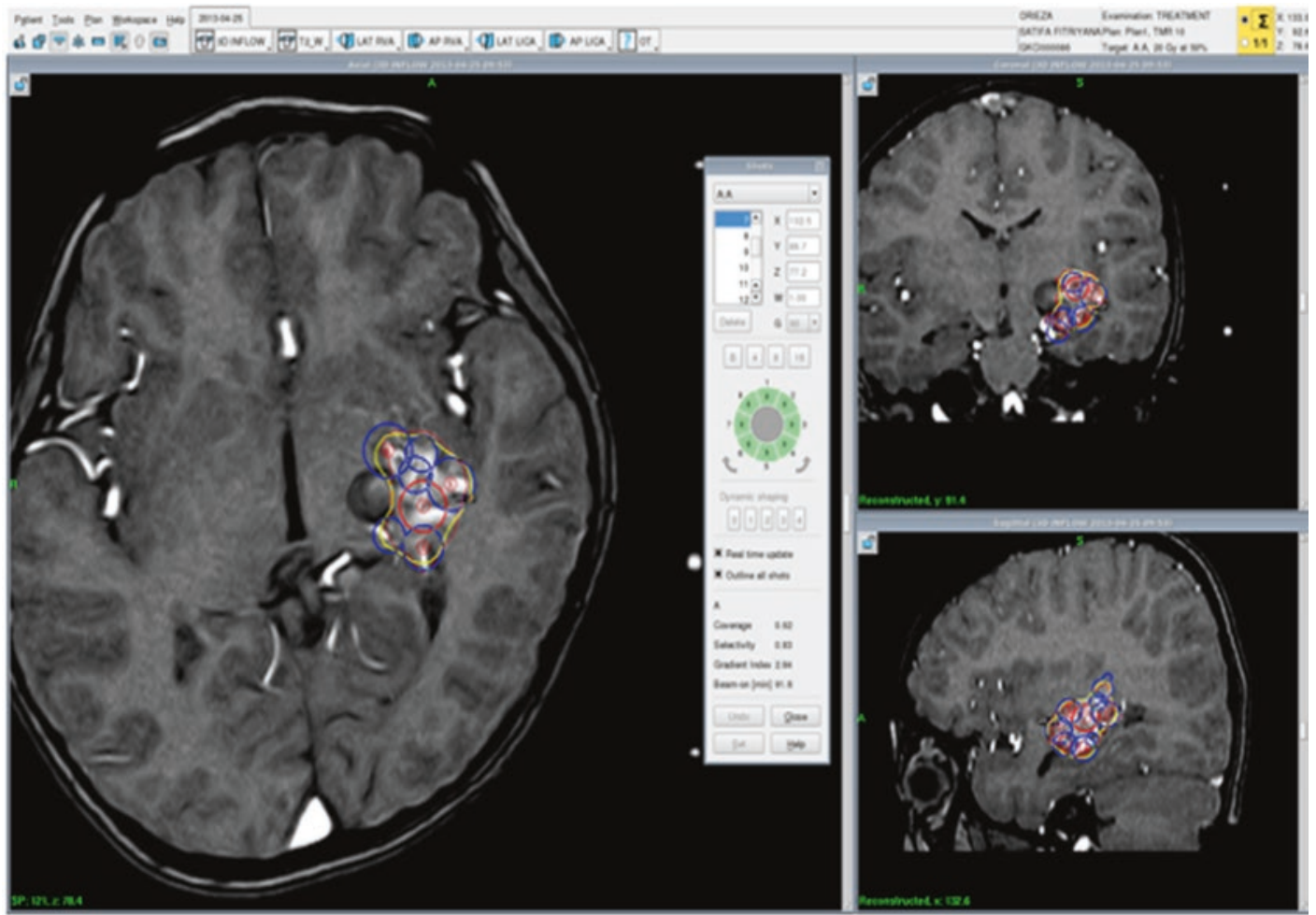

Fig. 32.7 Isocenter placement. Multiple isocenters (circles in blue line) are placed and arranged to form an isodose line (yellow line) conforming target lesion (red line) that has been previously delineated

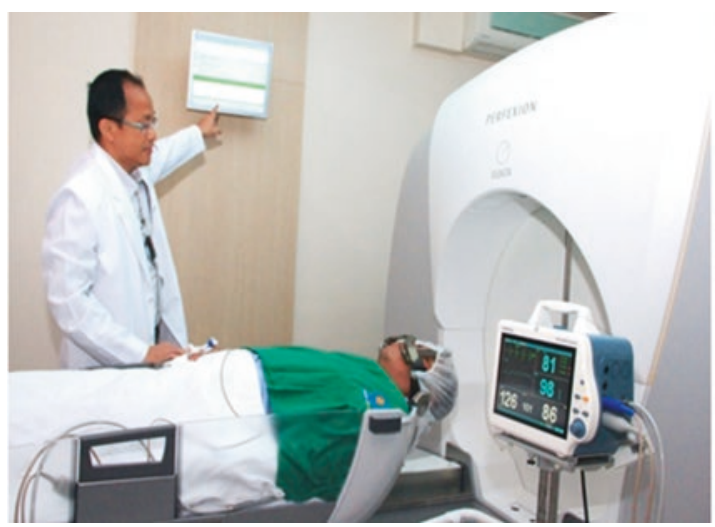

Fig. 32.8 Patient positioning and treatment delivery. Patient has been positioned, and frame is fixated; monitors for vital signs are attached, and treatment plan is rechecked

room, which is connected to control room as intercom, to monitor and to communicate with patients during treatment delivery.

When treatment is completed, patient is removed from treatment couch, and pins are

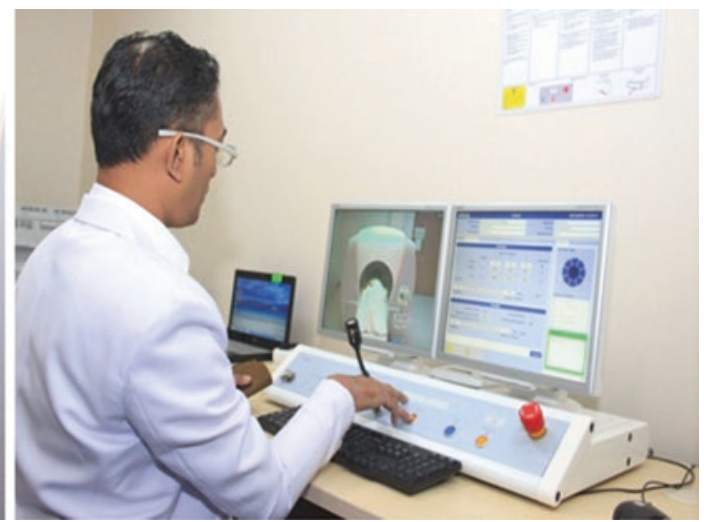

and ready (left). Treatment then can be initiated, and patient is monitored during the whole procedure through CCTV camera (right)

unscrewed during frame removal. Some patients may experience temporary headache after frame removal that dexamethasone and painkiller are routinely given. Finally, patients are observed for 1-2 $\mathrm{h}$ before discharge from hospital. 


\subsection{Surgeon Plan to Handle the Complication}

Complications after Gamma Knife surgery includes radiation-induced changes whether symptomatic or not, cyst formation, hemorrhage, and radiation-induced neoplasm. Postradiation image change, commonly known as adverse radiation effects (ARE), are the most common adverse effects following Gamma Knife surgery occurring in $33.8 \%$ of cases within 3-12 months. They can be symptomatic in $8.6 \%$ of patients but fortunately reversible in most, and only a small number of $1.8 \%$ is associated with permanent neurological deficit [4]. These changes are demonstrated by increased signal on T2-weighted or FLAIR MRI around nidus as shown in Fig. 32.9. Several mechanisms are proposed to be responsible for these changes including radiation injury to glial cells especially oligodendroglia resulting in demyelination, damage to endothelial cells with cytokine, and antigen release causing the disruption of blood-brain barrier with subsequent edema, generation of free radicals, auto immune response, and indirect effects through vascular insults with subsequent ischemia.

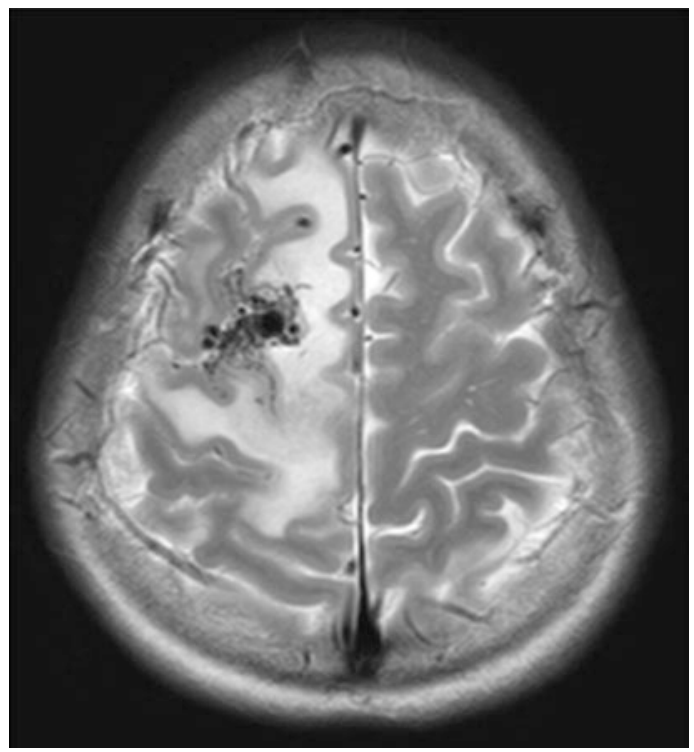

Fig. 32.9 Adverse radiation effects. Increased signal intensity on T2-weighted MRI around nidus
Many factors are related to these complications risk including larger nidus size, higher marginal dose, superficial and eloquent location, as well as less number of draining veins. Special care and considerations in treatment planning must be taken when factors related to increased risks such as larger nidus volume and eloquent locations are encountered. Lower marginal dose is typically given in these situations, and we would consider volume staging in large AVMs (nidus size above $4 \mathrm{~cm}$ or volume above $30 \mathrm{~cm}^{3}$ ).

Surprisingly, ARE is also considered a prognostic factor to complete obliteration. Those who have complete obliteration on long-term followup are more common to experience ARE starting earlier within 4-6 months, peaking within 7-12 months after Gamma Knife surgery, and to have more severe symptoms. Among this group of patients, ARE were evident in $74.4 \%$ and being significant in terms of symptom in $58.1 \%$ [5]. Steroids such as dexamethasone is commonly used to treat this condition.

Gamma Knife surgery doesn't increase risk of hemorrhage, compared to natural history of AVM. In fact, although debatable, it seems to reduce the risk especially when higher margin dose is administered [6, 7]. It is reasonable to have higher margin dose for small nidus, while for larger ones, dose should be lowered. Risk of cyst formation and neoplasia or malignancy on long-term follow-up is even far less. There are very few reports currently on neoplasia or malignancy induced by Gamma Knife surgery.

\subsection{Things to Be Observed and Postoperative Care/ Follow-Up}

Rarely we find excessive bleeding from pin site that we need to put stitches on. Some patients experience temporary headache or even being nauseous right after frame removal that we recommend observation at least an hour or two before discharge.

Patients are followed up clinically within 2 weeks posttreatment and every 6-month intervals subsequently within 3-year period. MRI 
examinations were scheduled within 6-12-month interval and DSA within 1-2-year interval. MRI is performed regularly to follow-up change and potential complications, while DSA is to confirm obliteration of AVM. For those who develop clinically significant ARE, intravenous steroid starting $10 \mathrm{mg}$ dexamethasone four times a day is initiated and soon is tapered off as clinical condition improves.

\section{References}

1. Abecassis IJ, Xu DS, Batjer HH, Bendok BR. Natural history of brain arteriovenous malformations: a systematic review. Neurosurg Focus. 2014;37(3):E7.

2. Gross BA, Du R. Natural history of cerebral arteriovenous malformations: a meta-analysis. J Neurosurg. 2013;118:437-43.
3. Starke RM, Yen C, Ding D, Sheehan JP. A practical grading scale for predicting outcome after radiosurgery for arteriovenous malformations: analysis of 1012 treated patients. J Neurosurg. 2013;119:981-7.

4. Yen C, Matsumoto JA, Wintermark M, Schwyzer L, Evans AJ, Jensen ME, et al. Radiation-induced imaging changes following gamma knife surgery for cerebral arteriovenous malformations. J Neurosurg. 2013;118:63-73.

5. Cohen-Inbar O, Lee C, Xu Z, Schlesinger D, Sheehan JP. A quantitative analysis of adverse radiation effects following gamma knife surgery for arteriovenous malformations. J Neurosurg. 2015;123:945-53.

6. Yen C, Sheehan JP, Schwyzer L, Schlesinger D. Hemorrhage risk of arteriovenous malformation before and during the latency period after gamma knife radiosurgery. Stroke. 2011;42:1691-6.

7. Pollock BE, Link MJ, Brown RD. The risk of stroke or clinical impairment after stereotactic radiosurgery for ARUBA-eligible patients. Stroke. 2013; 44:437-41.

Open Access This chapter is licensed under the terms of the Creative Commons Attribution 4.0 International License (http://creativecommons.org/licenses/by/4.0/), which permits use, sharing, adaptation, distribution and reproduction in any medium or format, as long as you give appropriate credit to the original author(s) and the source, provide a link to the Creative Commons license and indicate if changes were made.

The images or other third party material in this chapter are included in the chapter's Creative Commons license, unless indicated otherwise in a credit line to the material. If material is not included in the chapter's Creative Commons license and your intended use is not permitted by statutory regulation or exceeds the permitted use, you will need to obtain permission directly from the copyright holder. 